PROCEEDINGS OF THE

AMERICAN MATHEMATICAL SOCIETY

Volume 137, Number 7, July 2009, Pages 2273-2283

S 0002-9939(09)09867-0

Article electronically published on March 11, 2009

\title{
PROPER ACTIONS WHICH ARE NOT SATURATED
}

\author{
DAMIÁN MARELLI AND IAIN RAEBURN
}

(Communicated by Marius Junge)

\begin{abstract}
If a locally compact group $G$ acts properly on a locally compact space $X$, then the induced action on $C_{0}(X)$ is proper in the sense of Rieffel, with generalised fixed-point algebra $C_{0}(G \backslash X)$. Rieffel's theory then gives a Morita equivalence between $C_{0}(G \backslash X)$ and an ideal $I$ in the crossed product $C_{0}(X) \times G$; we identify $I$ by describing the primitive ideals which contain it, and we deduce that $I=C_{0}(X) \times G$ if and only if $G$ acts freely. We show that if a discrete group $G$ acts on a directed graph $E$ and every vertex of $E$ has a finite stabiliser, then the induced action $\alpha$ of $G$ on the graph $C^{*}$-algebra $C^{*}(E)$ is proper. When $G$ acts freely on $E$, the generalised fixed-point algebra $C^{*}(E)^{\alpha}$ is isomorphic to $C^{*}(G \backslash E)$ and Morita equivalent to $C^{*}(E) \times G$, in parallel with the situation for free and proper actions on spaces, but this parallel does not seem to give useful predictions for nonfree actions.
\end{abstract}

\section{INTRODUCTION}

A famous theorem of Green 2 asserts that if a locally compact group $G$ acts freely and properly on a locally compact space $X$, then the crossed product $C_{0}(X) \times$ $G$ is Morita equivalent to the algebra $C_{0}(G \backslash X)$ of continuous functions on the orbit space; a bimodule implementing the equivalence is obtained by completing $C_{c}(X)$ in the norm coming from a $C_{0}(G \backslash X)$-valued inner product (see [10]). Rieffel has considered a class of proper actions $\alpha: G \rightarrow$ Aut $A$ on noncommutative $C^{*}$-algebras which share key properties with the actions on $C_{0}(X)$ induced by proper actions on $X$, and for which there is a dense invariant subalgebra $A_{0}$ of $A$ with properties like those of the subalgebra $C_{c}(X)$ of $C_{0}(X)$ [12. Associated to every proper action is a Morita equivalence between an ideal $I_{Y}$ in the reduced crossed product $A \times_{\alpha, r} G$ and a generalised fixed-point algebra $A^{\alpha}$ contained in $M(A)$; a bimodule $Y$ implementing the equivalence is obtained by completing $A_{0}$ in a norm coming from an $A^{\alpha}$-valued inner product. Rieffel also abstracted the concept of freeness by identifying a class of saturated proper actions for which $I_{Y}=A \times_{\alpha, r} G$.

Rieffel's theory of proper actions has found substantial applications (see, for example, [11, 1, 3]), and there are many interesting examples of proper actions 12, 13, 6]. In particular, it was shown in [6] that if a discrete group $G$ acts freely on a directed graph $E$, then the induced action of $G$ on the graph algebra $C^{*}(E)$ is proper and saturated with generalised fixed-point algebra $C^{*}(G \backslash E)$ (and Rieffel's Morita

Received by the editors February 11, 2008.

2000 Mathematics Subject Classification. Primary 46L55.

This research was supported by the Australian Research Council through the ARC Centre for Complex Dynamic Systems and Control. 
equivalence was first obtained by Kumjian and Pask [5]). In all these applications, though, the emphasis is on the saturated proper actions which generalise free and proper actions on locally compact spaces.

Here we consider proper actions arising from nonfree actions on spaces and graphs. We had hoped to find useful descriptions of the generalised fixed-point algebra $A^{\alpha}$ and the ideal $I_{Y}$ which is Morita equivalent to it. We have succeeded when $G$ acts properly on a locally compact space $X$ : the induced action on $C_{0}(X)$ is proper with generalised fixed-point algebra $C_{0}(G \backslash X)$, and we can identify $I_{Y}$ by specifying the primitive ideals which contain it (see Theorem 3.3). We deduce that the action is saturated if and only if $G$ acts freely on $X$. For actions on graph algebras, the picture is less clear. We prove that an action of $G$ on a directed graph $E$ with finite stabilisers induces a proper action of $G$ on $C^{*}(E)$ (Theorem 4.1). However, the generalised fixed-point algebra may not be isomorphic to $C^{*}(G \backslash E)$, and while $I_{Y}$ can certainly be a proper ideal of $C^{*}(E) \times_{\alpha, r} G$, there are situations where the action on $E$ is not free but the induced action on $C^{*}(E)$ is saturated.

Our results on graph algebras may be a little surprising. Previous papers have exhibited a striking parallel between actions on spaces and actions on graphs: important Morita equivalences for free and proper transformation groups, such as Green's theorem and the symmetric imprimitivity theorem of [10, have direct analogues for free actions on directed graphs [5, 6. Our results suggest that this parallel completely breaks down in the absence of freeness.

We begin with a short section on preliminary material. In $₫ 3$, we consider actions of locally compact groups induced by proper actions on locally compact spaces. In \$4 we consider actions on graph algebras induced by actions on directed graphs with finite stabilisers. After proving that these are always proper, we discuss the question of identifying $C^{*}(E)^{\alpha}$ and $I_{Y}$; our main tool here is the dual graph construction, which allows us to use known results about free actions to study some nonfree actions.

\section{Preliminaries and notation}

Suppose $\alpha$ is an action of a locally compact group $G$ on a $C^{*}$-algebra $A$. The crossed product $A \times{ }_{\alpha} G$ is the completion of a convolution algebra $C_{c}(G, A)$ in a norm which makes $A \times{ }_{\alpha} G$ universal for covariant representations: every covariant representation $(\pi, U)$ of $(A, G, \alpha)$ on $\mathcal{H}$ has an integrated form $\pi \times U: A \times{ }_{\alpha} G \rightarrow$ $B(\mathcal{H})$ such that

$$
(\pi \times U(z) \xi \mid \eta)=\int_{G}\left(\pi(z(s)) U_{s} \xi \mid \eta\right) d s \text { for } z \in C_{c}(G, A) .
$$

The reduced crossed product $A \times_{\alpha, r} G$ is the completion of $C_{c}(G, A)$ in the norm determined by a single covariant representation $(\widetilde{\pi}, \lambda)$ induced from a faithful representation $\pi: A \rightarrow B\left(\mathcal{H}_{\pi}\right)$. (See [14, Chapter 2 and $\S 7.2$ ] for details.) We denote by $\hat{G}$ the set of irreducible unitary representations of a locally compact group $G$ and by 1 the trivial one-dimensional representation.

Following [12, Definition 1.2], we say that $\alpha: G \rightarrow$ Aut $A$ is proper with respect to $A_{0}$ if $A_{0}$ is a dense $\alpha$-invariant *-subalgebra of $A$ such that, for every $a, b \in A_{0}$,

(P1) the functions $s \mapsto a \alpha_{s}(b)$ and $s \mapsto \Delta(s)^{-1 / 2} a \alpha_{s}(b)$ are integrable, and 
(P2) there exists $\langle a, b\rangle_{D} \in M\left(A_{0}\right):=\left\{m \in M(A): c \in A_{0} \Longrightarrow m c \in A_{0}\right\}$ such that

$$
\langle a, b\rangle_{D} c=\int_{G} a \alpha_{s}\left(b^{*} c\right) d s \text { for every } c \in A_{0} .
$$

(Multipliers $\langle a, b\rangle_{D}$ with this property are automatically invariant under $\alpha$, as required in [12.) For such an action,

$$
A^{\alpha}:=\overline{\operatorname{span}}\left\{\langle a, b\rangle_{D}: a, b \in A_{0}\right\}
$$

is a $C^{*}$-subalgebra of $M(A)$ called the generalised fixed-point algebra, the set

$$
I_{Y}:=\overline{\operatorname{span}}\left\{{ }_{I}\langle a, b\rangle: s \mapsto \Delta(s)^{-1 / 2} a \alpha_{s}(b): a, b \in A_{0}\right\}
$$

is an ideal in the reduced crossed product $A \times_{\alpha, r} G$, and the closure $Y$ of $A_{0}$ in the norm $\|a\|^{2}:=\left\|\langle a, a\rangle_{D}\right\|$ is an $I_{Y^{-}} A^{\alpha}$ imprimitivity bimodule (see 12, Corollary 1.7]).

In 4 , we consider actions of a discrete group $G$ on the $C^{*}$-algebra of a rowfinite directed graph $E=\left(E^{0}, E^{1}, r, s\right)$. For graphs and their algebras we use the conventions of [8]. Thus, for example, a path in $E$ is a sequence $\mu=\mu_{1} \cdots \mu_{n}$ such that $s\left(\mu_{i}\right)=r\left(\mu_{i+1}\right)$, and the partial isometries in a Cuntz-Krieger $E$-family $\{S, P\}$ satisfy $S_{e}^{*} S_{e}=P_{s(e)}$ and $P_{v}=\sum_{r(e)=v} S_{e} S_{e}^{*}$. The $C^{*}$-algebra $C^{*}(E)$ is generated by a universal Cuntz-Krieger $E$-family $\{s, p\}$, and we write $\pi_{S, P}$ for the representation of $C^{*}(E)$ corresponding to a Cuntz-Krieger family $\{S, P\}$.

\section{Proper actions on locally compact spaces}

We consider a left action of a locally compact group $G$ on a locally compact space $X$ (always assumed to be Hausdorff). Throughout this section we assume that the action is proper, in the sense that inverse images of compact sets under the map $(s, x) \mapsto(x, s \cdot x): G \times X \rightarrow X \times X$ are compact. For such an action, the orbit space $G \backslash X$ is again locally compact and Hausdorff (see [14, Corollary 3.43]).

We now consider the induced action lt of $G$ on $C_{0}(X)$ defined by $\mathrm{lt}_{t}(f)(x):=$ $f\left(t^{-1} \cdot x\right)$. The following well-known fact is implicit in [12].

Proposition 3.1. The induced action lt of $G$ on $C_{0}(X)$ is proper with respect to the subalgebra $C_{c}(X)$, and the inclusion of $C_{0}(G \backslash X)$ in $C_{b}(X)=M\left(C_{0}(X)\right)$ is an isomorphism of $C_{0}(G \backslash X)$ onto the generalised fixed-point algebra $C_{0}(X)^{\mathrm{lt}}$.

Proof. For $f, g \in C_{0}(X)$, the functions $s \mapsto f \operatorname{lt}_{s}(g)$ are continuous and compactly supported, so they and their products with $\Delta(s)^{-1 / 2}$ are integrable; the function

$$
\langle f, g\rangle_{D}(G \cdot x):=\int_{G} \overline{f\left(t^{-1} \cdot x\right)} g\left(t^{-1} \cdot x\right) d t
$$

in $C_{c}(G \backslash X)$ defines a multiplier of $C_{0}(X)$ satisfying (P2). A compactness argument shows that every function in $C_{c}(G \backslash X)$ arises as $\langle f, g\rangle_{D}$, so the inclusion of $C_{0}(G \backslash X)$ in $C_{b}(X)=M\left(C_{0}(X)\right)$ is an isomorphism of $C_{0}(G \backslash X)$ onto $C_{0}(X)^{\mathrm{lt}}$.

The general theory of 12 implies that the ideal $I_{Y}$ defined in (2.2) is Morita equivalent to $C_{0}(G \backslash X)$. We now aim to identify $I_{Y}$ by describing the corresponding closed subset of the primitive ideal space of $C_{0}(X) \times_{\mathrm{lt}, r} G$. Since $G$ acts properly on $X, C_{0}(X) \times_{1 \mathrm{t}, r} G$ coincides with the full crossed product [7, Theorem 6.1], and we can define a family of irreducible representations of $C_{0}(X) \times_{\mathrm{lt}} G$ as follows. For $x \in X$ and each irreducible representation $V$ of $G_{x}:=\{s \in G: s \cdot x=x\}$, we consider the induced representation $\operatorname{Ind}_{G_{x}}^{G} V$ of $G$; we use the description of induced 
representations in [9, p. 296], which simplifies considerably in our situation because each $G_{x}$ is compact. Indeed, since the modular function $\Delta$ of $G$ is identically 1 on compact subgroups (the image of the subgroup is a compact subgroup of $(0, \infty)$ ), we can use the constant function 1 as the rho-function, and the measure $\alpha$ on $G / G_{x}$ is then $G$-invariant. So $\operatorname{Ind}_{G_{x}}^{G} V$ acts on the completion $\mathcal{H}_{x, V}$ of

$$
\mathcal{V}_{c}=\left\{\xi \in C_{c}\left(G, \mathcal{H}_{V}\right): \xi(r t)=V_{t}^{-1}(\xi(r)) \text { for } t \in G_{x}\right\}
$$

in the norm $\|\xi\|^{2}=\int_{G / G_{x}}\|\xi(r)\|^{2} d \alpha\left(r G_{x}\right)$, and $\operatorname{Ind}_{G_{x}}^{G} V$ is given by

$$
\left(\left(\operatorname{Ind}_{G_{x}}^{G} V\right)_{s} \xi\right)(t)=\xi\left(s^{-1} t\right) .
$$

There is a representation $\mu_{x}: C_{0}(X) \rightarrow B\left(\mathcal{H}_{x, V}\right)$ such that

$$
\left(\mu_{x}(f) \xi\right)(t)=f(t \cdot x) \xi(t) \text { for } \xi \in \mathcal{V}_{c},
$$

and then $\left(\mu_{x}, \operatorname{Ind}_{G_{x}}^{G} V\right)$ is a covariant representation of $\left(C_{0}(X), G, \mathrm{lt}\right)$.

Because $G \backslash X$ is Hausdorff, the assertions in the following proposition are contained in [14, 8.27 and 8.9]. However, in our setting we can give short direct proofs.

Proposition 3.2. For each $x \in X$ and $V \in \hat{G}_{x}$, the representation $\left(\mu_{x}, \operatorname{Ind}_{G_{x}}^{G} V\right)$ is irreducible, and every irreducible covariant representation of $\left(C_{0}(X), G, \mathrm{lt}\right)$ is equivalent to one of these.

Proof. First let $x \in X$ and $V \in \hat{G}_{x}$. Because $G$ acts properly, the map $t \mapsto t \cdot x$ induces a homeomorphism of $G / G_{x}$ onto $G \cdot x$ [14, Corollary 3.45], and $R_{G \cdot x}$ : $\left.f \mapsto f\right|_{G \cdot x}$ induces a surjection $R_{G \cdot x} \times$ id of $C_{0}(X) \times_{1 \mathrm{t}} G$ onto $C_{0}(G \cdot x) \times_{\mathrm{lt}} G \cong$ $C_{0}\left(G / G_{x}\right) \times_{1 \mathrm{t}} G$. Since $\mu_{x}$ vanishes on the kernel of $R_{G \cdot x}$, the integrated form $\mu_{x} \times \operatorname{Ind}_{G_{x}}^{G} V$ factors through $R_{G \cdot x} \times \mathrm{id}$, and the corresponding representation of $C_{0}\left(G / G_{x}\right) \times_{\mathrm{lt}} G$ is the representation $M \times \operatorname{Ind}_{G_{x}}^{G} V$ induced from the representation $V$ of $C^{*}\left(G_{x}\right)$ under Rieffel's Morita equivalence (see [9, Corollary C.31 and Theorem C.33]). Since induction by a Morita equivalence preserves irreducibility, $\mu_{x} \times \operatorname{Ind}_{G_{x}}^{G} V$ is irreducible.

Now suppose that $(\pi, U)$ is an irreducible representation of $\left(C_{0}(X), G\right.$, lt $)$. The representation $\pi$ is nondegenerate and hence extends to $M\left(C_{0}(X)\right)=C_{b}(X)$. For $f \in C_{0}(G \backslash X)$, covariance implies that $\pi(f)$ commutes with every $U_{t}$, and since it certainly commutes with every $\pi(g)$, irreducibility implies that $\pi(f) \in \mathbb{C} 1$. Thus there exists $G \cdot x \in G \backslash X$ such that $\pi(f)=f(G \cdot x) 1$ for every $f \in C_{0}(G \backslash X)$. If $a \in$ $C_{0}(X)$ satisfies $\left.a\right|_{G \cdot x}=0$, then we can approximate $a$ by $f a$ for some $f \in C_{0}(G \backslash X)$ satisfying $f(G \cdot x)=0$, and then $\pi(a) \sim \pi(f a)=f(G \cdot x) \pi(a)=0$. Thus $\pi \times U$ factors through $R_{G \cdot x} \times$ id and is given by a representation of $C_{0}\left(G / G_{x}\right) \times 1 \mathrm{t} G$; the imprimitivity theorem implies that this representation is equivalent to one induced from an irreducible representation $V$ of $G_{x}$, and pulling this equivalence back to $C_{0}(X) \times_{\mathrm{lt}} G$ shows that $(\pi, U)$ is equivalent to $\left(\mu_{x}, \operatorname{Ind}_{G_{x}}^{G} V\right)$.

Theorem 3.3. Suppose a locally compact group $G$ acts properly on a locally compact space $X$. Then $C_{0}(G \backslash X)$ is Morita equivalent to the ideal

$$
\bigcap\left\{\operatorname{ker}\left(\mu_{x} \times \operatorname{Ind}_{G_{x}}^{G} V\right): x \in X, V \in \hat{G}_{x}, V \neq 1\right\}
$$

in $C_{0}(X) \times_{1 \mathrm{t}} G$.

The crux of the proof is the following well-known lemma. 
Lemma 3.4. Suppose that $V$ is an irreducible representation of a compact group $K$ and $V \neq 1$. Then for every $h \in \mathcal{H}_{V}$, we have $\int_{K} V_{k} h d k=0$.

Proof. First suppose $\operatorname{dim} \mathcal{H}_{V}>1$ and $h \in \mathcal{H}_{V}$. Then $h_{0}:=\int_{K} V_{k} h d k$ is fixed under every $V_{l}$, and hence $\mathbb{C} h_{0}$ is an invariant subspace for $V$; since $\mathbb{C} h_{0}$ is not all of $\mathcal{H}_{V}$, it must be $\{0\}$, and $h_{0}=0$, as claimed. Now suppose $\operatorname{dim} \mathcal{H}_{V}=1$, so that $V: K \rightarrow \mathbb{T}$ is a character of $K$; since $V \neq 1$, there exists $l \in K$ such that $V_{l} \neq 1$. Then

$$
\int_{K} V_{k} d k=\int_{K} V_{k l} d k=\left(\int_{K} V_{k} d k\right) V_{l}
$$

forces $\int V_{k} d k=0$.

Proof of Theorem 3.3. We know from Rieffel's theory that $C_{0}(G \backslash X)$ is Morita equivalent to $I_{Y}$, so we want to identify $I_{Y}$ with the ideal (3.1). Since $I_{Y}$ is the intersection of the primitive ideals containing it, and since Proposition 3.2 implies that every primitive ideal has the form $\operatorname{ker}\left(\mu_{x} \times \operatorname{Ind}_{G_{x}}^{G} V\right)$, it suffices to show that

$$
I_{Y} \subset \operatorname{ker}\left(\mu_{x} \times \operatorname{Ind}_{G_{x}}^{G} V\right) \Longleftrightarrow V \neq 1 .
$$

Let $V$ be an irreducible representation of $G_{x}$, and write $(\mu, \lambda)$ for $\left(\mu_{x}, \operatorname{Ind}_{G_{x}}^{G} V\right)$. Let $a \in C_{c}\left(G, C_{0}(X)\right) \subset C_{0}(X) \times_{\mathrm{lt}} G$ and $\xi \in \mathcal{V}_{c}$; we need a formula for $\mu \times \lambda(a) \xi$. By expressing $(\mu \times \lambda(a) \xi \mid \eta)$ as in (2.1) and applying Fubini's theorem, we find that

$$
(\mu \times \lambda(a) \xi)(t)=\int_{G} a(s)(t \cdot x) \xi\left(s^{-1} t\right) d s \text { for } t \in G .
$$

Now we let $r=s^{-1} t$, decompose the Haar measure as in [9, Lemma C.2], and recall that the modular function is identically 1 on compact subgroups:

$$
\begin{aligned}
(\mu \times \lambda(a) \xi)(t) & =\int_{G} a\left(t r^{-1}\right)(t \cdot x) \xi(r) \Delta(r)^{-1} d r \\
& =\int_{G / G_{x}} \int_{G_{x}} a\left(t(u v)^{-1}\right)(t \cdot x) \xi(u v) \Delta(u v)^{-1} d v d \alpha\left(u G_{x}\right) \\
& =\int_{G / G_{x}} \Delta(u)^{-1} \int_{G_{x}} a\left(t(u v)^{-1}\right)(t \cdot x) V_{v}^{-1}(\xi(u)) d v d \alpha\left(u G_{x}\right) .
\end{aligned}
$$

Now we let $f, g \in C_{c}(X), a={ }_{I}\langle f, g\rangle$ and plug in

$$
a(r)(x)={ }_{I}\langle f, g\rangle(r)(x)=\Delta(r)^{-1 / 2} f(x) \overline{g\left(r^{-1} \cdot x\right)}
$$

to get

$$
\begin{aligned}
\left(\mu \times \lambda\left({ }_{I}\langle f, g\rangle\right) \xi\right)(t) & =\int_{G / G_{x}} \Delta(t u)^{-1 / 2} \int_{G_{x}} f(t \cdot x) \overline{g(u \cdot x)} V_{v}^{-1}(\xi(u)) d v d \alpha\left(u G_{x}\right) \\
& =\int_{G / G_{x}} \Delta(t u)^{-1 / 2} f(t \cdot x) \overline{g(u \cdot x)}\left(\int_{G_{x}} V_{v}^{-1}(\xi(u)) d v\right) d \alpha\left(u G_{x}\right) .
\end{aligned}
$$

If $V$ is not identically 1 , then Lemma 3.4 implies that the inside integral vanishes for every $u$, so $\mu_{x} \times \operatorname{Ind}_{G_{x}}^{G} V\left({ }_{I}\langle f, g\rangle\right)=0$ and

$$
I_{Y}:=\overline{\operatorname{span}}\left\{{ }_{I}\langle f, g\rangle\right\} \subset \operatorname{ker}\left(\mu_{x} \times \operatorname{Ind}_{G_{x}}^{G} V\right) .
$$

On the other hand, when $V=1, \mathcal{V}_{c}=C_{c}\left(G / G_{x}\right)$, the inside integral is $\xi\left(u \cdot G_{x}\right)$, and

$$
\left(\mu \times \lambda\left({ }_{E}\langle f, g\rangle\right) \xi\right)(t)=\int_{G / G_{x}} \Delta(t u)^{-1 / 2} f(t \cdot x) \overline{g(u \cdot x)} \xi\left(u \cdot G_{x}\right) d \alpha\left(u G_{x}\right) ;
$$


taking $\xi, f$ and $g$ to be positive functions with $\xi\left(G_{x}\right)>0, f(x)>0$ and $g(x)>0$ shows that this need not vanish, so $I_{Y}$ is not contained in $\operatorname{ker}\left(\mu_{x} \times \operatorname{Ind}_{G_{x}}^{G} V\right)$.

Corollary 3.5. Suppose a locally compact group $G$ acts properly on a locally compact space $X$. Then the action lt on $C_{0}(X)$ is saturated (in the sense that $\left.I_{Y}=C_{0}(X) \times_{1 \mathrm{t}} G\right)$ if and only if $G$ acts freely on $X$.

Proof. It is proved in 10, for example, that the action is saturated when $G$ acts freely. If $G$ does not act freely, then there is a point $x$ whose stabiliser $G_{x}$ has $\left|G_{x}\right|>1$. Then $G_{x}$ has an irreducible representation $V$ which is not identically 1, and then $\mu_{x} \times \operatorname{Ind}_{G_{x}}^{G} V$ is an irreducible representation of $C_{0}(X) \times_{\text {lt }} G$ whose kernel is a proper ideal. So (3.2) implies that $I_{Y}$ is not all of $C_{0}(X) \times_{1 \mathrm{t}} G$.

Example 3.6. Consider the action of the two-element group $C_{2}=\{e, a\}$ on $\mathbb{T}$ by reflection in the $x$-axis (so $a \cdot e^{\pi i \theta}=e^{-\pi i \theta}$ ). For $\theta \in[0,1]$ and $f \in C(\mathbb{T})$, we define

$$
\pi_{\theta}(f):=\left(\begin{array}{cc}
f\left(e^{\pi i \theta}\right) & 0 \\
0 & f\left(e^{-\pi i \theta}\right)
\end{array}\right), \quad U_{a}:=\left(\begin{array}{ll}
0 & 1 \\
1 & 0
\end{array}\right) .
$$

It is easy to check that $\left(\pi_{\theta}, U\right)$ is a covariant representation of $\left(C(\mathbb{T}), C_{2}\right.$, lt $)$. For $\theta \in(0,1)$, the ranges of $\pi_{\theta}$ and $U$ generate $M_{2}(\mathbb{C})$; for $\theta=0$ or $\theta=1, \pi_{\theta}(f)$ is a multiple of the identity matrix $1_{2}$, and $C^{*}\left(\pi_{\theta}(C(\mathbb{T})), U\right)=C^{*}\left(U_{a}\right)$, which is the $C^{*}$-algebraic direct sum $\mathbb{C} P_{1} \oplus \mathbb{C} P_{-1}$ associated to the spectral projections

$$
P_{1}=2^{-1}\left(1+U_{a}\right)=2^{-1}\left(\begin{array}{ll}
1 & 1 \\
1 & 1
\end{array}\right) \quad \text { and } \quad P_{-1}=2^{-1}\left(1-U_{a}\right)=2^{-1}\left(\begin{array}{cc}
1 & -1 \\
-1 & 1
\end{array}\right) .
$$

The point is that when $\theta$ is 0 or 1 , the stabiliser of $e^{\pi i \theta}$ is $C_{2}$, and there are two one-dimensional irreducible representations $\left(\mu_{e^{\pi i \theta}}, V\right)$. The representations in the invariant subspaces $\mathbb{C} P_{1}$ and $\mathbb{C} P_{-1}$ are equivalent to $\left(\mu_{e^{\pi i \theta}}, V\right)$ for $V_{a}=1$ and $V_{a}=-1$, respectively. So

$$
I_{Y} \cong\left\{f \in C\left([0,1], M_{2}(\mathbb{C})\right): f(0) \text { and } f(1) \in \mathbb{C} P_{1}\right\} .
$$

Conjugating by $W:=2^{-1 / 2}\left(\begin{array}{cc}1 & 1 \\ 1 & -1\end{array}\right)$ diagonalises $U$, carries $C(\mathbb{T}) \times_{1 \mathrm{t}} C_{2}$ into

$$
A:=\left\{f \in C\left([0,1], M_{2}\right): f(0) \text { and } f(1) \text { are diagonal }\right\},
$$

and $I_{Y}$ into $\left\{f \in A: f(0)_{22}=f(1)_{22}=0\right\}$. Notice that the spectrum $\hat{I}_{Y}$ is homeomorphic to $[0,1]$, which is what we would expect since $C_{2} \backslash \mathbb{T}$ is homeomorphic to $[0,1]$. The algebra $A$, on the other hand, has highly non-Hausdorff spectrum: $\pi_{\theta} \times U$ converges to two distinct points of $\hat{A}$ as $\theta \rightarrow 0+$ and as $\theta \rightarrow 1-$ (as in [9, Example A.25]).

\section{Proper actions on graph algebras}

We suppose that a (discrete) group $G$ acts on the left of a row-finite directed graph $E$ and that the stabiliser $G_{v}$ of each vertex $v$ in $E$ is finite. As in [5], there is an action $\alpha: G \rightarrow \operatorname{Aut} C^{*}(E)$ such that $\alpha_{t}\left(s_{\mu} s_{\nu}^{*}\right)=s_{t \cdot \mu} s_{t \cdot \nu}^{*}$ for all $\mu, \nu \in E^{*}$.

Theorem 4.1. Suppose that $G$ acts on $E$ with finite stabilisers. Then $\left(C^{*}(E), \alpha\right)$ is proper with respect to the dense subalgebra

$$
Y_{0}(E):=\operatorname{span}\left\{s_{\mu} s_{\nu}^{*}: \mu, \nu \in E^{*}\right\} .
$$


The characterising property of $\alpha$ implies that $Y_{0}(E)$ is $\alpha$-invariant. By linearity, it suffices to verify property (P1) for $a=s_{\alpha} s_{\beta}^{*}$ and $b=s_{\mu} s_{\nu}^{*}$. But then

$$
a \alpha_{t}(b) \neq 0 \Longrightarrow s_{\beta}^{*} s_{t \cdot \mu} \neq 0 \Longrightarrow r(\beta)=t \cdot r(\mu)
$$

which is true for only finitely many $t$; thus the function $t \mapsto a \alpha_{t}(b)$ has finite support, and (P1) is trivially true. To verify property (P2), we need the following lemma.

Lemma 4.2. Suppose $b \in Y_{0}(E)$. Then there exists $\Sigma_{G}(b) \in M\left(Y_{0}(E)\right)$ such that

$$
\Sigma_{G}(b) a=\sum_{t \in G} \alpha_{t}(b) a \text { and } a \Sigma_{G}(b)=\sum_{t \in G} a \alpha_{t}(b) \quad \text { for every } a \in Y_{0}(E)
$$

Again, it suffices to prove Lemma 4.2 for $b=s_{\mu} s_{\nu}^{*}$, and we need an estimate:

Lemma 4.3. Suppose that $F$ is a finite subset of $G$. Then

$$
\left\|\sum_{t \in F} s_{t \cdot \mu} s_{t \cdot \nu}^{*}\right\| \leq \sqrt{\left|G_{r(\mu)}\right|\left|G_{r(\nu)}\right|}
$$

Proof. We choose a faithful representation $\pi_{S, P}: C^{*}(E) \rightarrow B(\mathcal{H})$, and let $h \in \mathcal{H}$. Pythagoras's theorem gives

$$
\begin{aligned}
\left\|\sum_{t \in F} S_{t \cdot \mu} S_{t \cdot \nu}^{*} h\right\|^{2} & =\left\|\sum_{w \in E^{0}} \sum_{t \in F, r(t \cdot \mu)=w} S_{t \cdot \mu} S_{t \cdot \nu}^{*} h\right\|^{2} \\
& =\sum_{w \in E^{0}}\left\|\sum_{t \in F, t \cdot r(\mu)=w} S_{t \cdot \mu} S_{t \cdot \nu}^{*} P_{t \cdot r(\nu)} h\right\|^{2} .
\end{aligned}
$$

For fixed $w \in E^{0}$ we have

$$
\begin{aligned}
\left\|\sum_{t \in F, t \cdot r(\mu)=w} S_{t \cdot \mu} S_{t \cdot \nu}^{*} P_{t \cdot r(\nu)} h\right\|^{2} \leq\left(\sum_{t \in F, t \cdot r(\mu)=w}\left\|P_{t \cdot r(\nu)} h\right\|\right)^{2} \\
=\sum_{t_{i} \in F, t_{1} \cdot r(\mu)=t_{2} \cdot r(\mu)=w}\left\|P_{t_{1} \cdot r(\nu)} h\right\|\left\|P_{t_{2} \cdot r(\nu)} h\right\| \\
\leq \sum_{t_{i} \in F, t_{1} \cdot r(\mu)=t_{2} \cdot r(\mu)=w} 2^{-1}\left(\left\|P_{t_{1} \cdot r(\nu)} h\right\|^{2}+\left\|P_{t_{2} \cdot r(\nu)} h\right\|^{2}\right) .
\end{aligned}
$$

Since $\{t: t \cdot v=s \cdot v\}=s G_{v}$ has $\left|G_{v}\right|$ elements, for $w, v \in E^{0}$ and $i=1,2$ we have

$$
\left|\left\{t_{i} \in F: t_{i} \cdot r(\mu)=w\right\}\right| \leq|\{t \in G: t \cdot r(\mu)=w\}| \leq\left|G_{r(\mu)}\right|
$$

So

$$
\begin{aligned}
\sum_{t_{i} \in F, t_{1} \cdot r(\mu)=t_{2} \cdot r(\mu)=w} 2^{-1}\left(\left\|P_{t_{1} \cdot r(\nu)} h\right\|^{2}+\left\|P_{t_{2} \cdot r(\nu)} h\right\|^{2}\right) & \\
& \leq \sum_{t \in F, t \cdot r(\mu)=w}\left|G_{r(\mu)}\right|\left\|P_{t \cdot r(\nu)} h\right\|^{2} .
\end{aligned}
$$


Adding up over $w$ gives

$$
\begin{aligned}
\left\|\sum_{t \in F} S_{t \cdot \mu} S_{t \cdot \nu}^{*} h\right\|^{2} & \leq\left|G_{r(\mu)}\right| \sum_{w \in E^{0}} \sum_{t \in F, t \cdot r(\mu)=w}\left\|P_{t \cdot r(\nu)} h\right\|^{2} \\
& =\left|G_{r(\mu)}\right| \sum_{t \in F}\left\|P_{t \cdot r(\nu)} h\right\|^{2} \\
& =\left|G_{r(\mu)}\right| \sum_{v \in E^{0}} \sum_{t \in F, t \cdot r(\nu)=v}\left\|P_{v} h\right\|^{2} \\
& \leq\left|G_{r(\mu)}\right| \sum_{v \in E^{0}}\left|G_{r(\nu)}\right|\left\|P_{v} h\right\|^{2} \\
& =\left|G_{r(\mu)}\right|\left|G_{r(\nu)}\right|\|h\|^{2} .
\end{aligned}
$$

Since $\pi_{S, P}$ is isometric, the result follows.

Proof of Lemma 4.2. For $b=s_{\mu} s_{\nu}^{*}$, we define $L_{b}, R_{b}: Y_{0}(E) \rightarrow Y_{0}(E)$ by

$$
L_{b}(a)=\sum_{t \in G} \alpha_{t}\left(s_{\mu} s_{\nu}^{*}\right) a=\sum_{t \in G} s_{t \cdot \mu} s_{t \cdot \nu}^{*} a \text { and } R_{b}(a)=\sum_{t \in G} a s_{t \cdot \mu} s_{t \cdot \nu}^{*} ;
$$

we know from (4.1) that for fixed $a$, all these summands vanish outside a finite set $F$, and then Lemma 4.3 gives the estimate

$$
\left\|L_{b}(a)\right\| \leq\left\|\sum_{t \in F} s_{t \cdot \mu} s_{t \cdot \nu}^{*}\right\|\|a\| \leq \sqrt{\left|G_{r(\mu)}\right|\left|G_{r(\nu)}\right|}\|a\|
$$

with constant independent of $a$. Thus $L_{b}$ extends to a bounded linear operator $L_{b}: C^{*}(E) \rightarrow C^{*}(E)$; so does $R_{b}$. A calculation shows that $R_{b}(a) c=a L_{b}(c)$ for $a, c \in Y_{0}(E)$, and this extends to $a, c \in C^{*}(E)$ by continuity. So the pair $\left(L_{b}, R_{b}\right)$ is a double centraliser of $C^{*}(E)$, and hence defines a multiplier $\Sigma_{G}(b) \in M\left(C^{*}(E)\right)$. Since left and right multiplication by $\Sigma_{G}(b)$ (that is, the maps $L_{b}$ and $R_{b}$ ) map $Y_{0}(E)$ into $Y_{0}(E), \Sigma_{G}(b)$ belongs to $M\left(Y_{0}(E)\right)$.

To check that $\left(C^{*}(E), \alpha\right)$ satisfies property $(\mathrm{P} 2)$, we take $\langle b, c\rangle_{D}:=\Sigma_{G}\left(b^{*} c\right)$, and this completes the proof of Theorem 4.1 .

Theorem 4.1 and Corollary 1.7 of $\left[12\right.$ imply that the closure $Y(E)$ of $Y_{0}(E)$ in the $D$-norm implements a Morita equivalence between $C^{*}(E)^{\alpha}$ and an ideal $I_{Y(E)}$ in $C^{*}(E) \times_{\alpha, r} G$. Theorem 1.6 of [6] tells us that when $G$ acts freely, $C^{*}(E)^{\alpha}$ is isomorphic to the algebra $C^{*}(G \backslash E)$ of the quotient graph, and $I_{Y(E)}$ is all of $C^{*}(E) \times_{\alpha, r} G$. We will use this result and the dual graph construction to get information about $C^{*}(E)^{\alpha}$ and $I_{Y(E)}$ for nonfree actions which are free on some path space $E^{n}$.

The dual graph $\hat{E}$ is the quadruple $\left(E^{1}, E^{2}, r, s\right)$ in which $r(f e)=f$ and $s(f e)=$ $e$. Assume that $E$ has no sources. The proof of [8, Corollary 2.6], for example, shows that there is an isomorphism $\phi$ of $C^{*}(\hat{E})=C^{*}(t, q)$ onto $C^{*}(E)=C^{*}(s, p)$ such that

$$
\phi\left(t_{f e}\right)=s_{f} s_{e} s_{e}^{*} \text { and } \phi\left(q_{e}\right)=s_{e} s_{e}^{*} .
$$

The action of $G$ on $E$ induces an action on $\hat{E}$ such that $t \cdot(f e)=(t \cdot f)(t \cdot e)$. This action on $\hat{E}$ also has finite stabilisers, because the stabiliser of any edge in $E$ is contained in the stabiliser of its range. Thus Theorem 4.1 implies that the induced action $\hat{\alpha}$ of $G$ on $C^{*}(\hat{E})$ is proper with respect to the subalgebra $Y_{0}(\hat{E})$. 
The isomorphism $\phi$ satisfies $\phi \circ \hat{\alpha}=\alpha \circ \phi$, and hence by [14, Theorem 2.48] induces an isomorphism $\phi \times$ id of $C^{*}(\hat{E}) \times{ }_{\hat{\alpha}} G$ onto $C^{*}(E) \times{ }_{\alpha} G$, which maps the dense subalgebra $C_{c}\left(G, C^{*}(\hat{E})\right)$ into $C_{c}\left(G, C^{*}(E)\right)$ according to the formula

$$
(\phi \times \mathrm{id})(z)(t)=\phi(z(t))
$$

Composing with $\phi \times$ id carries the regular representation induced from a faithful representation $\pi$ of $C^{*}(E)$ into the regular representation induced from $\pi \circ \phi$, and hence $\phi \times$ id descends to an isomorphism $\phi \times{ }_{r}$ id of $C^{*}(\hat{E}) \times{ }_{\hat{\alpha}, r} G$ onto $C^{*}(E) \times{ }_{\alpha, r} G$.

Proposition 4.4. Suppose that $E$ has no sources and that $G$ acts on $E$ with finite stabilisers. Let $\phi: C^{*}(\hat{E}) \rightarrow C^{*}(E)$ be the isomorphism characterised by (4.2). Then $\bar{\phi}$ restricts to an isomorphism of $C^{*}(\hat{E})^{\hat{\alpha}}$ onto $C^{*}(E)^{\alpha}$, and the triple

$$
\left(\phi \times_{r} \text { id, } \phi_{Y}, \bar{\phi} \mid\right):\left(I_{Y(\hat{E})}, Y(\hat{E}), C^{*}(\hat{E})^{\hat{\alpha}}\right) \rightarrow\left(I_{Y(E)}, Y(E), C^{*}(E)^{\alpha}\right)
$$

is an isomorphism of imprimitivity bimodules.

Lemma 4.5. The isomorphism $\phi$ characterised by (4.2) maps $Y_{0}(\hat{E})$ onto $Y_{0}(E)$, and its extension $\bar{\phi}: M\left(C^{*}(\hat{E})\right) \rightarrow M\left(C^{*}(E)\right)$ satisfies $\bar{\phi} \circ \Sigma_{G}^{\hat{E}}=\Sigma_{G}^{E} \circ \phi$.

Proof. We first take $\sigma \in \hat{E}^{n}$ and $\tau \in \hat{E}^{m}$ with $s(\sigma)=s(\tau)$, and aim to show that $\phi\left(t_{\sigma} t_{\tau}^{*}\right)$ is in $Y_{0}(E)$. Every path in $\hat{E}^{n}$ has the form $\hat{\mu}:=\left(\mu_{1} \mu_{2}\right)\left(\mu_{2} \mu_{3}\right) \cdots\left(\mu_{n} \mu_{n+1}\right)$ for some $\mu \in E^{n+1}$, so there exist $\mu \in E^{n+1}$ and $\nu \in E^{m+1}$ such that $\sigma=\hat{\mu}$ and $\tau=\hat{\nu}$. Then $s(\sigma)=s(\tau)$ implies that $\mu_{n+1}=\nu_{m+1}$, and we can compute

$$
\begin{aligned}
\phi\left(t_{\sigma} t_{\tau}^{*}\right) & =\left(s_{\mu_{1}} s_{\mu_{2}} s_{\mu_{2}}^{*}\right)\left(s_{\mu_{2}} s_{\mu_{3}} s_{\mu_{3}}^{*}\right) \cdots\left(s_{\mu_{n}} s_{\mu_{n+1}} s_{\mu_{n+1}}^{*}\right)\left(s_{\nu_{m}+1} s_{\nu_{m+1}}^{*} s_{\nu_{m}}^{*}\right) \cdots\left(s_{\nu_{2}} s_{\nu_{2}}^{*} s_{\nu_{1}}^{*}\right) \\
& =s_{\mu_{1}}\left(s_{\mu_{2}} s_{\mu_{2}}^{*} s_{\mu_{2}}\right) \cdots\left(s_{\mu_{n}} s_{\mu_{n}}^{*} s_{\mu_{n}}\right)\left(s_{\mu_{n+1}} s_{\mu_{n+1}}^{*} s_{\nu_{m+1}} s_{\nu_{m+1}}^{*}\right)\left(s_{\nu_{m}}^{*} s_{\nu_{m}} s_{\nu_{m}}^{*}\right) \cdots s_{\nu_{1}}^{*} \\
& =s_{\mu_{1}} s_{\mu_{2}} \cdots s_{\mu_{n}} s_{\mu_{n+1}} s_{\nu_{m+1}}^{*} s_{\nu_{m}}^{*} \cdots s_{\nu_{1}}^{*} \\
& =s_{\mu} s_{\nu}^{*}
\end{aligned}
$$

which certainly belongs to $Y_{0}(E)$. Since $E$ has no sources, the elements $s_{\mu} s_{\nu}^{*}$ with $|\mu| \geq 1$ and $|\nu| \geq 1$ span $Y_{0}(E)$, and the same computation shows that every spanning element $s_{\mu} s_{\nu}^{*}=\phi\left(s_{\hat{\mu}} s_{\hat{\nu}}^{*}\right)$ belongs to the range of $\phi$. Thus $\phi$ maps $Y_{0}(\hat{E})$ onto $Y_{0}(E)$.

The extension $\bar{\phi}$ is characterised by $\bar{\phi}(m) \phi(b)=\phi(m b)$ for $m \in M\left(C^{*}(\hat{E})\right)$ and $b \in C^{*}(\hat{E})$, and it suffices by continuity to consider $b \in Y_{0}(\hat{E})$. Then for $a \in Y_{0}(\hat{E})$,

$$
\bar{\phi}\left(\Sigma_{G}^{\hat{E}}(a)\right) \phi(b)=\phi\left(\sum_{t \in G} \hat{\alpha}_{t}(a) b\right)=\sum_{t \in G} \alpha_{t}(\phi(a)) \phi(b)=\Sigma_{G}^{E}(\phi(a)) \phi(b)
$$

implies that $\bar{\phi} \circ \Sigma_{G}^{\hat{E}}=\Sigma_{G}^{E} \circ \phi$.

Proof of Proposition 4.4. For $b, c \in Y_{0}(\hat{E})$, the last assertion in Lemma 4.5 implies

$$
\bar{\phi}\left(\langle b, c\rangle_{D}\right)=\bar{\phi} \circ \Sigma_{G}^{\hat{E}}\left(b^{*} c\right)=\Sigma_{G}^{E}\left(\phi\left(b^{*} c\right)\right)=\langle\phi(b), \phi(c)\rangle_{D} .
$$

Equation (4.4) implies, first, that $\bar{\phi}$ maps $C^{*}(\hat{E})^{\hat{\alpha}}$ into $C^{*}(E)^{\alpha}$, and, second, since $\phi$ maps $Y_{0}(\hat{E})$ onto $Y_{0}(E)$, that $\bar{\phi}$ maps $C^{*}(\hat{E})^{\hat{\alpha}}$ onto $C^{*}(E)^{\alpha}$. Since $\phi$ is isometric on $C^{*}(\hat{E})$, (4.4) also implies that $\phi: Y_{0}(\hat{E}) \rightarrow Y_{0}(E)$ is isometric for the inner-product norms, and hence extends to an isometry $\phi_{Y}: Y(\hat{E}) \rightarrow Y(E)$. Equation (4.4) now extends to elements $b, c$ of the completion $Y(\hat{E})$, and says that $\phi_{Y}$ is a $\bar{\phi} \mid$ compatible isomorphism of Hilbert modules. This isomorphism $\phi_{Y}$ in turn induces an isomorphism $\phi_{\mathcal{K}}$ of $I_{Y(\hat{E})}=\mathcal{K}(Y(\hat{E}))$ onto $I_{Y(E)}=\mathcal{K}(Y(E))$ such that

$$
\phi_{\mathcal{K}}\left({ }_{I}\langle b, c\rangle\right)={ }_{I}\left\langle\phi_{Y}(b), \phi_{Y}(c)\right\rangle \text {. }
$$


However, for $b, c \in Y_{0}(\hat{E})$, a computation using (4.3) shows that

$$
(\phi \times \mathrm{id})\left({ }_{I}\langle b, c\rangle\right)(t)={ }_{I}\langle\phi(b), \phi(c)\rangle(t) \text { for every } t \in G ;
$$

this implies, first, that $\phi \times_{r}$ id $=\phi_{\mathcal{K}}$, and hence is an isomorphism of $I_{Y(\hat{E})}$ onto $I_{Y(E)}$, and, second, that $\left(\phi \times_{r} \mathrm{id}, \phi_{Y}, \bar{\phi} \mid\right)$ has the algebraic properties required of an imprimitivity-bimodule isomorphism.

Corollary 4.6. Suppose that $E$ has no sources and that the action of $G$ on $E$ is free on $E^{1}$. Then $I_{Y(E)}=C^{*}(E) \times{ }_{\alpha} G$, and $C^{*}(E)^{\alpha}$ is isomorphic to $C^{*}(G \backslash \hat{E})$.

Proof. Since $G$ acts freely on $E^{1}=\hat{E}^{0}$, [6. Theorem 1.6] implies that $I_{Y(\hat{E})}=$ $C^{*}(\hat{E}) \times_{\hat{\alpha}, r} G$ and $C^{*}(\hat{E})^{\hat{\alpha}}$ is isomorphic to $C^{*}(G \backslash \hat{E})$. Since [4, Corollary 3.3] implies that $C^{*}(\hat{E}) \times_{\hat{\alpha}, r} G=C^{*}(\hat{E}) \times_{\hat{\alpha}} G$, the result follows from Proposition 4.4.

Example 4.7. Let $E$ be the Cuntz graph consisting of one vertex $v$ and two loops $f, g$ at $v$, so that $C^{*}(E)$ is the Cuntz algebra $\mathcal{O}_{2}$. The cyclic group $C_{2}=\{e, a\}$ acts on $E$ by fixing $v$ and swapping $f$ and $g: a \cdot f=g$ and $a \cdot g=f$. Since $C_{2}$ acts freely on $E^{1}$, Corollary 4.6 implies that $I_{Y(E)}=\mathcal{O}_{2} \times{ }_{\alpha} C_{2}$ and that $\mathcal{O}_{2}^{\alpha}$ is isomorphic to $C^{*}\left(C_{2} \backslash \hat{E}\right)$. Here $\hat{E}$ is the complete graph on two vertices; the quotient $C_{2} \backslash \hat{E}$ has the single vertex $C_{2} \cdot f=C_{2} \cdot g$ and two edges $C_{2} \cdot f f$ and $C_{2} \cdot f g$, and hence is isomorphic to $E$. Thus Rieffel's theorem gives a Morita equivalence between $\mathcal{O}_{2} \times{ }_{\alpha} C_{2}$ and $\mathcal{O}_{2}$.

This example shows that $C^{*}(E)^{\alpha}$ is not always isomorphic to $C^{*}(G \backslash E)$ : the quotient graph $C_{2} \backslash E$ consists of the single loop $C_{2} \cdot f$ based at $C_{2} \cdot v$, and $C^{*}\left(C_{2} \backslash E\right)$ is isomorphic to $C(\mathbb{T})$, which is quite different from $C^{*}(E)^{\alpha} \cong \mathcal{O}_{2}$.

We can apply Corollary 4.6 to $\hat{E}$, whose dual is isomorphic to $E_{1,2}:=\left(E^{2}, E^{1}\right.$, $r, s)$, and then inductively to the higher-order duals $E_{n, n+1}:=\left(E^{n+1}, E^{n}, r, s\right)$ in which $r(\mu)=\mu_{1} \cdots \mu_{n}$ and $s(\mu)=\mu_{2} \cdots \mu_{n+1}$. If $G$ acts freely on $E^{n}$, say, then $n-1$ applications of Proposition 4.4 show that $I_{Y(E)}$ is all of $C^{*}(E) \times{ }_{\alpha} G$ and that the generalised fixed-point algebra is isomorphic to $C^{*}\left(G \backslash E^{n}\right)$.

These arguments do not apply to every action with finite stabilisers. For example, if $f$ is a loop in $E$ which is fixed by $t \in G$, then the path $f^{n}:=f f \cdots f$ ( $n$ times) is a vertex in $E_{n, n+1}$ which is fixed by $t$. So it is not clear to us whether we can make sensible conjectures about arbitrary actions with finite stabilisers. The ideal $I_{Y(E)}$ can certainly be proper - indeed, when a finite group $G$ acts trivially on $E, I_{Y(E)}$ is the subalgebra of $C\left(G, C^{*}(E)\right) \subset C^{*}(E) \times{ }_{\alpha} G=C^{*}(E) \times{ }_{\alpha, r} G$ consisting of the constant functions, which is easily seen to be isomorphic to $C^{*}(E)$, and Rieffel's equivalence becomes tautological. On the other hand, it does seem likely that the full and reduced crossed products of $\left(C^{*}(E), G, \alpha\right)$ will still coincide when the stabilisers are finite, even though the indirect arguments used in 4, Corollary 3.3] or [6, Corollary 3.12] will not work when the action is not free.

\section{REFERENCES}

[1] B. Abadie, Generalised fixed-point algebras of certain actions on crossed products, Pacific J. Math. 171 (1995), 1-21. MR:1362977 (96m:46121)

[2] P. Green, $C^{*}$-algebras of transformation groups with smooth orbit space, Pacific J. Math. 72 (1977), 71-97. MR0453917(56:12170)

[3] A. an Huef and I. Raeburn, Mansfield's imprimitivity theorem for arbitrary closed subgroups, Proc. Amer. Math. Soc. 132 (2004), 1153-1162. MR2045432(2005b:46116) 
[4] S. Kaliszewski, J. Quigg and I. Raeburn, Skew products and crossed products by coactions, J. Operator Theory 46 (2001), 411-433. MR1870415 (2003a:46096)

[5] A. Kumjian and D. Pask, $C^{*}$-algebras of directed graphs and group actions, Ergodic Theory Dynam. Systems 19 (1999), 1503-1519. MR1738948 (2000m:46125)

[6] D. Pask and I. Raeburn, Symmetric imprimitivity theorems for graph $C^{*}$-algebras, Internat. J. Math. 12 (2001), 609-623. MR1843869 (2002g:46114)

[7] N.C. Phillips, Equivariant K-Theory for Proper Actions, Pitman Research Notes in Math., vol. 178, Longman, Harlow, copublished in U.S. with Wiley, New York, 1989. MR991566 (90g:46105)

[8] I. Raeburn, Graph Algebras, CBMS Regional Conference Series in Math., vol. 103, Amer. Math. Soc., Providence, RI, 2005. MR2135030(2005k:46141)

[9] I. Raeburn and D.P. Williams, Morita Equivalence and Continuous-Trace $C^{*}$-Algebras, Math. Surveys and Monographs, vol. 60, Amer. Math. Soc., Providence, RI, 1998. MR 1634408 (2000c:46108)

[10] M.A. Rieffel, Applications of strong Morita equivalence to transformation group $C^{*}$-algebras, Operator Algebras and Applications, Proc. Symp. Pure Math., vol. 38, Part I, Amer. Math. Soc., Providence, RI, 1982, pages 299-310. MR679709 (84k:46046)

[11] M.A. Rieffel, Deformation quantization of Heisenberg manifolds, Comm. Math. Phys. 122 (1989), 531-562. MR1002830 (90e:46060)

[12] M.A. Rieffel, Proper actions of groups on $C^{*}$-algebras, Mappings of Operator Algebras, Progress in Math., vol. 84, Birkhäuser, Boston, 1990, pages 141-182. MR1103376 (92i:46079)

[13] M.A. Rieffel, Integrable and proper actions on $C^{*}$-algebras, and square-integrable representations of groups, Expositiones Math. 22 (2004), 1-53. MR2166968 (2006g:46108)

[14] D.P. Williams, Crossed Products of $C^{*}$-Algebras, Math. Surveys and Monographs, vol. 134, Amer. Math. Soc., Providence, RI, 2007. MR2288954 (2007m:46003)

ARC Centre for Complex Dynamic Systems and Control, University of Newcastle, NSW 2308, Australia

E-mail address: damian.marelli@newcastle.edu.au

School of Mathematics and Applied Statistics, University of Wollongong, NSW 2522, Australia

E-mail address: raeburn@uow.edu.au 